\title{
Monads, Indexes and Transformations
}

\author{
Françoise Bellegarde ${ }^{\star}$ and James Hook ${ }^{\star \star}$ \\ Pacific Software Research Center \\ Oregon Graduate Institute of Science \& Technology \\ 19600 N.W. von Neumann Drive \\ Beaverton, OR 97006-1999 \\ \{bellegar, hook\}ocse.ogi.edu
}

\begin{abstract}
The specification and derivation of substitution for the de Bruijn representation of $\lambda$-terms is used to illustrate programming with a functionsequence monad. The resulting program is improved by interactive program transformation methods into an efficient implementation that uses primilive machine arithmetic. These transformations illustrate new techniques that assist the discovery of the arithmetic structure of the solution.
\end{abstract}

\section{Introduction}

Substitution is one of many problens in computer science that, once understood in one context, is understood in all contexts. Why, then, must a different substitution function be written for every abstract syntax implemented? This paper shows how to specify substitution once and use the monadic structure of the specification to instantiate it on different abstract syntax structures. It also shows how to interactively derive an efficient implementation of substitution from this very abstract specification.

Formal methods that support reasoning about free algebras from first principles based on their inductive structure are theoretically attractive because they have simple and expressive theories. However, in practice they often lead to ineflicient algorithms because they fail to exploit the "algebras" implemented in computer hardware. This paper examines this problem by giving a systematic program development and then describing a series of (potentially) automatic program transformations that may be used to achieve an efficient implementation.

The particular program development style employed is based on the categorical notion of a monad. This approach to specification has been advocated by Wadler[8] and is strongly influenced by Moggi's work on semantics[6]. The substitution algorithm for $\lambda$-calculus terms represented with de Bruijn indexes serves as the primary example. The development of the specification is a refinement of an example in llook, Kieburtz and Sheard[5]. It is noteworthy because a non-standard category is used; the earlier work did not identify this category.

The specification is transformed into first-order equations using techniques implemented in the partial evaluator Schism[4]. It is then refined to an equivalent first-

\footnotetext{
* Bellegarde is currently at Western Washington University, Bellingham, WA 98225.

** Both authors are supported in part by a grant from the NSF (CCR-9101721).
} 
The first thing to observe about the sequence is that its general shape is $\sigma_{i+1} 0=0$ and $\sigma_{i+1}(n+1) \approx \sigma_{i} n$. To make it exact it is necessary to increment all global variables in $\sigma_{i} n$ without incrementing the local variables. This is done by another sequence of functions:

$$
\begin{aligned}
& f_{0} n=n+1 \quad f_{1} 0=0 \quad f_{2} 0=0 \\
& f_{1}(n+1)=n+2 \quad f_{2} 1=1 \\
& f_{2}(n+2)=n+3
\end{aligned}
$$

Observe that in the example a single application of $f_{1}$ to the body of $\sigma_{1} 1$ accounts for $\lambda .01$ being adjusted to $\lambda .02$. In general the $f_{i}$ are generated by $f_{i+1} 0=0$ and $f_{i+1}(n+1)=\left(f_{i} n\right)+1$. So, assuming a map that applies a family of functions, the family of substitution functions, $\left(\sigma_{0}, \sigma_{1}, \ldots\right)$, is given by the initial substitution, $\sigma_{0}$, and the recurrence $\sigma_{i+1} 0=0$ and $\sigma_{i+1}(n+1)=\operatorname{map}\left(f_{0}, f_{1}, \ldots\right)\left(\sigma_{i} n\right)$. Given the sequence of functions, $\left(\sigma_{0}, \sigma_{1}, \ldots\right)$, mapping indexes to terms, the map function for sequences can be used to apply the sequence of substitution functions. This, however, results in terms of terms, since every variable has replaced its index by a term. This is not a problem, however, because the Term type constructor developed below is designed to be a monad; monads have a polymorphic function, mult, which performs the requisite fattening.

\section{Monads}

A monad is a concept from category theory that has been used to provide structure to semantics[6] and to specifications[8]. In the computer science setting a monad is defined by a parametric data type constructor, $T$, and three polymorphic functions: map $:(\alpha \rightarrow \beta) \rightarrow T \alpha \rightarrow T \beta$, unit $: \alpha \rightarrow T \alpha$, and mull :TT $\alpha \rightarrow T \alpha$. The map function is required to satisfy ${\text { map } i d_{\alpha}}=i d_{T \alpha}$ and map $(f \circ g)=$ map $f \circ$ map $g$. The polymorphic functions unit and mult must satisfy mult ${ }_{\alpha} \circ$ unit $_{T_{\alpha}}=i d_{T \alpha}$, mult $_{\alpha} \circ$ (map unit $t_{\alpha}=i d_{T \alpha}$ and $m u l_{\alpha} \circ$ mull $_{T \alpha}=m u l_{\alpha} \circ\left(\right.$ map mult m $_{\alpha}$. A simple example of a monad is list. For lists, map is the familiar mapcar function of Lisp, unit is the function that produces a singleton list, and mull is the concatenate function that flattens a list of lists into a single list. Other examples of monads are given by Wadler[8].

Several categorical concepts are implicit above. The functional programming category has types as objects and (computable) functions as arrows. (Values are viewed as constant functions--arrows from the one element type.) The requirements on map specify that the type constructor $T$ and the map function together define a functor. The polymorphic types of unit and mull implicitly require them to be natural transformations. The three laws given for them are the monad laws.

Monads have been used to structure specifications (and semantics) because it is often possible to characterize interesting facets of a specification as a monad. Algorithms to exploit the particular facet may frequently be expressed in terms of the map, unit and mult functions with no explicit details of the type constructors. Finally, the many facets are brought together by composing the type constructors. 


\section{The Term Monad}

The development in Sect. 1 suggests that the specification of the substitution operation will be straightforward in a monadic data type with an appropriate map. To be monadic, the data type must be parametric. The following simple type declaration is sufficient ${ }^{2}$ :

$$
\begin{aligned}
\text { datatype } \operatorname{Term}(\alpha)= & \operatorname{Var}(\alpha) \\
& \mid \operatorname{Abs}(\operatorname{Term}(\alpha)) \\
& \mid \operatorname{App}(\operatorname{Term}(\alpha) * \operatorname{Term}(\alpha))
\end{aligned}
$$

Using techniques developed in earlier work, it is possible to automatically generate map, mull and unit functions for this type realizing a monadic structure[5]. Unfortunately, the map function obtained with those techniques does not work with families of functions.

To accommodate the function sequences a new category, FUNSEQ, is used. The objects are data types, as before, but the morphisms are sequences of functions (formally $\operatorname{Hom}(A, B)=\left(B^{A}\right)^{\omega}$ ). Identities are constant sequences of identities from the underlying category; composition is pointwise, i.e. $\left(f_{i}\right)_{i \in \omega} \circ\left(g_{i}\right)_{i \in \omega}=\left(f_{i} \circ g_{i}\right)_{i \in \omega}$.

The map function for Term exploits the new structure by shifting the series of functions whenever it enters a new context. Its definition is given as a functional program:

$$
\begin{aligned}
& \operatorname{map}\left(f_{0}, f_{1}, \ldots\right)(\operatorname{Var} x)=\operatorname{Var}\left(\left(f_{0}, f_{1}, \ldots\right) x\right) \\
& \operatorname{map}\left(f_{0}, f_{1}, \ldots\right)(\text { Abst })=A b s\left(\operatorname{map}\left(f_{1}, f_{2}, \ldots\right) t\right) \\
& \operatorname{map}\left(f_{0}, f_{1}, \ldots\right)\left(A p p\left(t, t^{\prime}\right)\right)=A p p\left(\operatorname{map}\left(f_{0}, f_{1}, \ldots\right) t, \operatorname{map}\left(f_{0}, f_{1}, \ldots\right) t^{\prime}\right)
\end{aligned}
$$

It is easily verified that (Term, map) satisfy the categorical definition of a functor.

Looking at these definitions, it is clear how to insert an ordinary function or value into the category, and it is straightforward to insert the families of functions needed for the example by giving the initial element of the sequence and the functional that generates all others. However, it is also necessary to define the mapping that pulls a computation from FUNSEQ back into the category of functional programs. This is accomplished by taking the first element of the function sequence. Thus, one way to realize the map function of FUNSEQ in a functional programming setting is with the map_with_policy function introduced in Hook, Kieburtz and Sheard[5]:

$$
\begin{aligned}
\text { map_with_policy } Z f(\operatorname{Var} x) & =\operatorname{Var}(f x) \\
\text { map_with_policy } Z f(A b s t) & =A b s(\text { map_with_policy } Z(Z f) t) \\
\text { map_with_policy } Z f\left(A p p\left(l, t^{\prime}\right)\right)= & A p p(\text { map_with_policy } Z f t, \\
& \text { map_with_policy } \left.Z f t^{\prime}\right)
\end{aligned}
$$

In this encoding $Z$ is the functional that generates the sequence and $f$ is the seed value. That is, $\left(\operatorname{map}\left(f, Z f, Z^{2} f, \ldots\right)\right)_{0}=$ map_with_policy $Z f$. Note the projection

\footnotetext{
2 This is a simplified form of the Term data type in Hook, Kieburtz and Sheard[5]. An anonymous referee has pointed ont that an alternative structure can be used instead. The argument to $A b s$ may be given the type $\operatorname{Tcrm}(1+\alpha)$ (where + is interpreted as a discriminated union). While this structure is very interesting, it is not possible to express the map function for this type in the Standard ML type system. Preliminary results indicate this structure can be used to specify substitution.
} 
of the first element from the family of functions on the left hand side indicated by the subscript 0 .

The unit and mult functions automatically generated for Term can be lifted to FunSeq. Simple inductions show that they satisfy the monad laws.

With these definitions in place the complete definition of substitution is given in Fig. 1. Note that the algorithm makes no explicit mention of the data constructors. It only uses the information about the type implicit in the definition of map_with_policy, unit and mult.

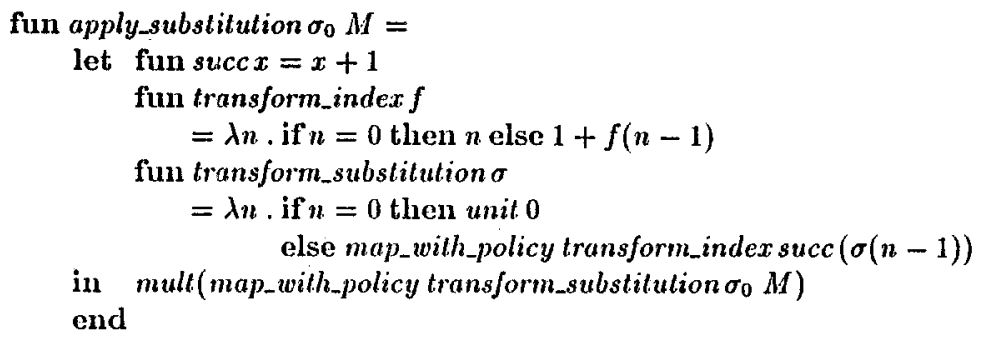

Fig. 1. Substitution function

\section{Transformation to a First-Order Set of Equations}

To obtain a practical algorithm, the substitution function apply_substitution in Fig. 1 must be made more efficient. This section shows how this transformation can be done automatically. Program transformation systems operate on systems of firstorder equations. To apply them to the specification of substitution the higher-order facets must be translated into first-order structures. A partial evaluation system is used to accomplish this.

The software allowing a complete automatic transformation is not yet written. The transformations below have been performed with the Schism partial evaluator [4] and the Astre program transformation system [1], which are not yet integrated and do not use the same language.

\subsection{Transformation of the map_with_policy Operator}

The first step is to rewrite the program using the map_with_policy operator for the type $\operatorname{Term}(\alpha)$ as a system of first-order functions. A partial evaluator can be used to specialize higher-order functions decreasing their order level. For example, consider the particular function $\sigma_{0}$ in the example in Sect. 1, and the call apply_substitution $\sigma_{0}$. A partial evaluator produces a program that does not contain apply_substitution in its full generality; it specializes the definition of apply_substitution 
for the particular constant $\sigma_{0}$. This specialization, called apply_substitution_$\sigma_{0}$, does not have a function as an argument, so it is first-order.

Unfortunately, this technique is insufficient for processing calls of map_with_policy, which is called twice in the program in Fig. 1. The specialization of map_with_policy for a particular policy function $K$ and seed function $g_{0}$ gives the following function $M w p_{-} g$ :

$$
\begin{aligned}
& M w p_{-} g(g, \operatorname{Var}(n))=\operatorname{Var}(g(n)) \\
& M w p_{-} g(g, A b s(t))=A b s\left(M w p_{-} g(K g, t)\right) \\
& M w p_{-} g\left(g, A p p\left(t, t^{\prime}\right)\right)=A p p\left(M w p_{-} g(g, t), M w p_{-} g\left(g, t^{\prime}\right)\right)
\end{aligned}
$$

The funclion Mwp-g has a function as an argument. But if it is specialized for a particular function $g_{0}$, the partial evaluator has to specialize the internal call $M w p_{-} g(K g, t)$; it loops on this attempt. Fortunately, the partial evaluator is able to detect this circumstance, allowing it to select another technique. The alternative technique translates the higher-order functions into a system of first-order functions. This standard encoding, which is due to Reynolds [7], is outlined below.

1. The first step constructs a data type that encodes how the higher-order arguments are manipulated and applied. In this case the functions to be encoded are $g_{0}$ and $K g$. For the constant function, $g_{0}$, a constant $C$ is introduced as a summand in the data type Func. The argument $K g$ cannot be encoded by a simple constant value because it contains $g$ as a free variable. Since $g$ is a higher-order parameter, it will already be represented by a value of type Func. Hence the new constructor, $F$, representing the application of $K$, must have type $F u n c \rightarrow$ Func. This gives the data type Func, defined datatype Func $=C \mid F(F u n c)$. . The introduction of this type is a rediscovery of the sequence of functions $g_{0}, g_{1}, \ldots$ because it encodes each function in the family. The function $g_{0}$ is encoded by $C$, and the function $g_{3}$, for example, is encoded by $F(F(F(C))$ ), which is written $F^{3}$.

2. The functions appearing as actual arguments are replaced by their encodings. The argument functions do not exist anymore-they are replaced by first-order data. In the call $M w p_{-} g\left(g_{0}, M\right), g_{0}$ is no longer a function but a first-order value, $\left\lceil g_{0}\right\rceil$, of type Func. The definition of $M w p_{-} g$ leads to the new function $M w p_{-} g$ :

$$
\begin{array}{ll}
M w p_{-} g^{\prime}(\lceil g\rceil, \operatorname{Var}(n)) & =\operatorname{Var}(\lceil g\rceil(n)) \\
M w p_{-} g^{\prime}(\lceil g\rceil, A b s(t)) & =A b s\left(M w p_{-} g^{\prime}(F(\lceil g\rceil), t)\right) \\
M w p_{-} g^{\prime}\left(\lceil g\rceil, A p p\left(t, t^{\prime}\right)\right) & =A p p\left(M w p_{-} g^{\prime}(\lceil g\rceil, t), M w p_{-} g^{\prime}\left(\lceil g\rceil, t^{\prime}\right)\right)
\end{array}
$$

But since $\lceil g\rceil$ is not a function, the application $\lceil g\rceil(n)$ is nonsense.

3. To make sense of the applications of functional parameters in the original programs "application" functions are introduced. Specifically the function apply_g, defined below, decodes applications of the form $\lceil g\rceil(n)$.

$$
\begin{aligned}
& a p p l y_{-} g(C, n)=g_{0}(n) \\
& a p p l y-g(F(\lceil g\rceil), n)=(K \lambda n . a p p l y-g(\lceil g\rceil, n))(n) \text {. }
\end{aligned}
$$

Note that apply-g/ is a first-order function because its argument, $\lceil g\rceil$, is an element of the type $F$ unc. The partial evaluator unfolds the definition of the policy 
function $K$ to get a first-order expression of apply $g(F([g]), n)$. The definition of $M w p_{-} g^{\prime}$ can be completed into:

$$
\begin{array}{ll}
M w p_{-} g^{\prime}(\lceil g\rceil, \operatorname{Var}(n)) & =\operatorname{Var}(\operatorname{apply} g(\lceil g\rceil, n)) \\
M w p_{-} g^{\prime}([g], A b s(t)) & =A b s\left(M w p_{-} g^{\prime}(F(\lceil g\rceil), t)\right) \\
M w p_{-} g^{\prime}\left(\lceil g\rceil, A p p\left(t, t^{\prime}\right)\right) & =A p p\left(M w p_{-} g^{\prime}(\lceil g\rceil t), M w p_{-} g^{\prime}\left(\lceil g\rceil, t^{\prime}\right)\right)
\end{array}
$$

Recall that this encoding is done with respect to a specific call of map_with_policy $Z g_{0} M$. In the program in Fig. 1 there are two such calls. If the partial evaluator succeeds in the transformation of (4), then the new functions corresponding to $M w_{-} g$ and $a p p l y_{-} g$ will constitute a first-order program equivalent to the funclions generated by map_wilh_policy. This step of the transformation can be automated using a partial evaluator.

\subsection{Application to apply_subslitution.}

Using the preceding techniques, the function apply_substitution is successfully transformed into the first-order program in Fig. 2. The data type Subst and the data type Fseq are introduced using the techniques above for the encodings of transform_index and transform.substitution.

$$
\begin{array}{rr}
\text { datatype } S u b s t=S 0 & \text { datatype Fseq }=S U C C \\
& \mid \text { SUBST(Subst.) }
\end{array}
$$

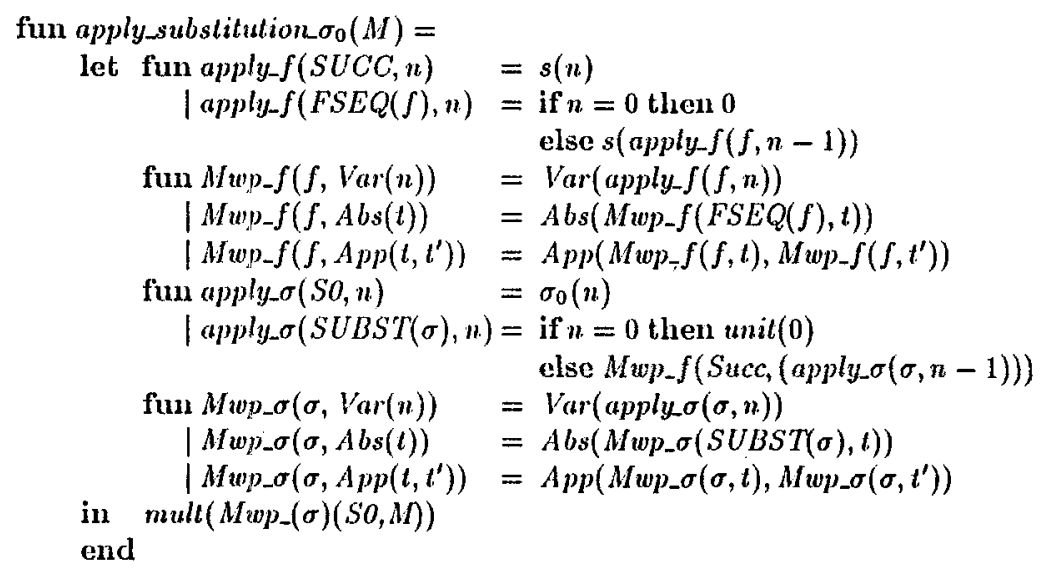

Fig. 2. First-order Program

These two data types are isomorphic to the data type $\mathrm{Nat}^{3}$ which is implemented efficiently in the hardware. However, the specialized function $M w p_{-} \sigma$ does not exploit

\footnotetext{
${ }^{3}$ The constructors for the data type $N a t$ are 0 and $s$, i.e. datatype $N a t=0 \mid s(N a t)$.
} 
the efficient implementation since it uses the (essentially unary) representation of the data type instead. Thus, the function apply_ $\sigma$ must peel ofl all of the data constructors each time $M w p_{-} \sigma$ is applied to $\operatorname{Var}(n)$. For example, after three levels of abstraction, $\sigma_{3}$ is represented by $\operatorname{SUBST}(\operatorname{SUBST}(S U B S T(S 0)))$. (The same is also true of the function $M w p_{-} f$.) To eliminate this inefficiency, which was present in the calling belsavior of the original specification, the data types Subst and Fseq must be changed to the uniform data type $\mathrm{Nal}$. This transformation can be performed automatically by Astre. Ultimately the explicit use of $\mathrm{Nal}$ will facilitate the use of primitive arithmetic in the program.

\section{Simple Transformations}

The following two simple transformations are performed automatically by Astre after introducing new function symbols. The first one introduces indexes to count the level of abstractions. The second replaces the composition of $M w p$ with the function mult by a single function. The order of these transformations does not matter; they can be done simultaneously.

For technical reasons recursive definitions of the form $g(n)=$ if $n=0$ then $e_{1} \mathrm{else}_{2}$ are manipulated more effectively by $\Lambda$ stre in the equivalent form $g(0)=\varepsilon_{1}[0 / n]$ and $g(s(n))=c_{2}[s(n) / n]$. The notation $c\left[e^{\prime} / x\right]$ denotes the substitution of expression $e^{\prime}$ for $x$ in $e$. This restriction of the form of equations ensures the termination of the rewriting used by Astre to unfold the definition of $g$.

\subsection{Introduction of Indexes}

The isomorphism between the automatically generated type Subst and the natural numbers is made explicit by introducing the function iso_ $\sigma: N a t \rightarrow S u b s t:$

$$
\begin{gathered}
\text { fun fun iso_ } \sigma(s(i))=S U B S T(\text { iso } \sigma(i)) \\
\mid i s o_{-} \sigma(0)=S 0
\end{gathered}
$$

The functions apply_ $\sigma$ and $M w p_{-} \sigma$ are replaced by the new functions $\sigma(i, n)$ (for $\left.\sigma_{i}(n)\right)$ and $M w p_{-} \sigma^{\prime}$, respectively. These functions satisfy $\sigma(i, n)=a p p l y \_\sigma\left(i s o_{-} \sigma(i), n\right)$ and $M w p_{-} \sigma^{\prime}(i, n)=M w p_{-} \sigma($ iso_ $\sigma(i), n)$. Using these new equations, the Astre system implements the dala lype $S u b s t$ using the dala type $N a t$. New functions to implement the data type Fscq using Nat are also provided to the Astre system which then gives the program in Fig. 3. The program in Fig. 3 does not improve the performance of the program in Fig. 2. However, its explicit use of numbers is key to the improvements presented in the next section.

\subsection{Composition Step}

The transformation continues with a simple (automatic) step that replaces the composition of mull with $M w p-\sigma^{\prime}$ by a single function. ${ }^{4}$ This is accomplished by introducing a function symbol, Ewp, which is equated to the composition of mult with

\footnotetext{
This composition is oftell called the Kleisli star or nalural cxtension. Eup is a mnemonic for extension with policy.
} 


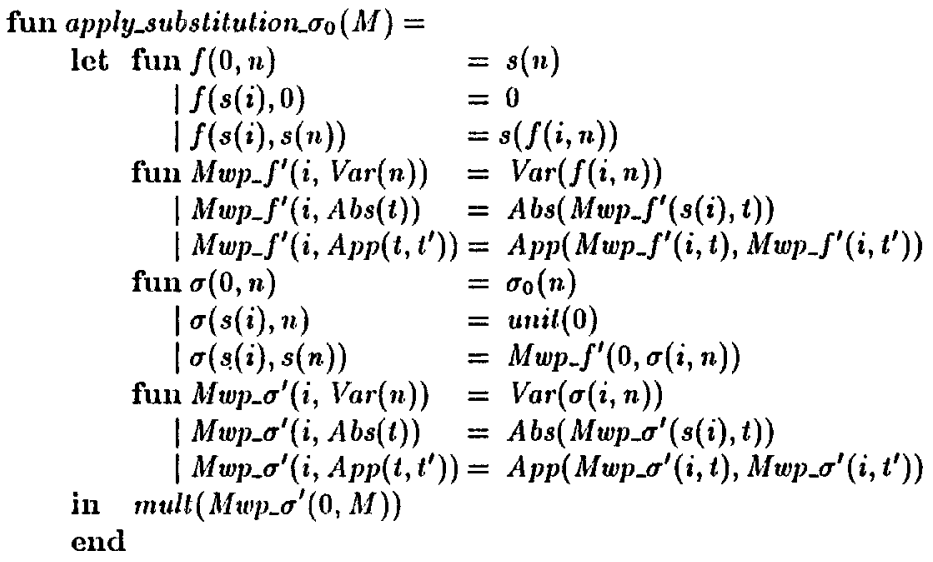

Fig. 3. Program will indexes

$M w p_{-} \sigma^{\prime}$, i.e., $\operatorname{Evpp}(0, M)=m u l t\left(M w p_{-} \sigma^{\prime}(0, M)\right)$. Astre gives a program which uses neither mull, nor $M w p \_\sigma^{\prime}$ that includes the following definition of Ewp:

$$
\text { fun } \begin{aligned}
E w p(i, \operatorname{Var}(n)) & =\sigma(i, n) \\
\mid \operatorname{Ewp}(i, A b s(t)) & =A b s(\operatorname{Ewp}(s(i), t)) \\
\mid E w p\left(i, A p p\left(t, t^{\prime}\right)\right) & =A p p\left(E w p(i, t), \operatorname{Ewp}\left(i, t^{\prime}\right)\right)
\end{aligned}
$$

The main body of the function is then replaced by $\operatorname{Ewp}(0, M)$. The functions mult and $M w p_{-} \sigma^{\prime}$, which have become useless, are removed. Since the $M w p_{-} \sigma^{\prime}$ has now been eliminated, $M w p_{-} f^{\prime}$ is renamed $M w p$ to simplify the nomenclature below.

\section{Transformation of the Sequence of the $\sigma$ Functions}

The transformations in this section exploit the arithmetic arguments introduced above to replace the expensive and redundant recursive calculations in $\sigma$ and $E w p$ with index arithmetic.

The function $\sigma(i, n)$ of the transformed program is a rediscovery of the series of functions $\sigma_{i}(n)$ of Sect. 1. To further refine this program a specific instance of apply_substitution $\sigma_{0}$ must be specified. In what follows, the substitution function $\sigma_{0}$, needed for the contraction described in Sect. 1, is used to illustrate the specialization. Recall that $\sigma_{0}$ replaces variables of index 0 with the term $\lambda .01$, which is represented by $A b s(A p p(\operatorname{Var}(0), \operatorname{Var}(1)))$. Thus, $\sigma_{0}(0)=A b s(A p p(\operatorname{Var}(0), \operatorname{Var}(1)))$ and $\sigma_{0}(s(n))=\operatorname{unit}(n)$. Unfolding these equations yields a complete definition of $\sigma(i, n)$ :

$$
\begin{aligned}
& \sigma(0,0)=A b s(A p p(\operatorname{Var}(0), \operatorname{Var}(1))) \\
& \sigma(0, s(n))=\operatorname{unit}(n) \\
& \sigma(s(i), 0)=u n i t(0) \\
& \sigma(s(i), s(n))=\operatorname{Mwp}(0, \sigma(i, n))
\end{aligned}
$$


Since the equational program is complete with respect to $N a t * N a t$, the computation of any instance of $\sigma(i, n)$ results in a ground constructor term. For example, $\sigma(4,2)$ yields:

$$
\begin{aligned}
\sigma(s(s(s(s(0)))), s(s(0))) & \rightarrow \\
M w p(0, \sigma(s(s(s(0))), s(0))) & \rightarrow \\
M w p(0, M w p(0, \sigma(s(s(0)), 0))) & \rightarrow{ }^{*} \operatorname{Var}(s(s(0)))
\end{aligned}
$$

Rewrites (6) and (7) are unfoldings by equation (5). Computation of any instance of $\sigma(i, n)$ by naturals can begin with unfoldings using (5) until a subterm, $\sigma(u, v)$, in which $u$ and/or $v$ are equal to 0 is obtained.

This suggests a target program of the form:

$$
\sigma(i, n)=\text { if } i>n \text { then } e_{1} \text { else if } i=n \text { then } e_{2} \text { else } e_{3}
$$

where $e_{1}, e_{2}$, and $c_{3}$ are expressions. The transformation will be beneficial if these expressions are efficient. This step introduces a form of function definition by a conditional (instead of structural induction) that violates the technical restriction on programs used to assure termination of rewriting as required by the Astre system. Presently, Astre does not perform this part of the transformation. Moreover, the transformation does not directly generate the conditional; instead it generates the complete definition: $\sigma(s(i)+k, k)=u_{1}, \sigma(k, k)=u_{2}$ and $\sigma(k, s(n)+k)=u_{3}$.

\subsection{First Transformation Step}

The general strategy of the two transformation steps that follow is to discover arithmetic operations implicit in the recursion structure of programs. The first step in this process is a definition that makes the iteration structure of functions explicit.

Definition 1. Let $x$ be a variable of type $\alpha$, let $y_{i}$ be a term of type $\beta_{i}$ for each $i=1, \cdots, n$, and let $\varphi$ be a function of type $\beta_{1} * \cdots * \alpha * \cdots * \beta_{n} \rightarrow \alpha$. The function $\hat{\varphi}$ of type $N a l *\left(\beta_{1} * \cdots * \alpha * \cdots * \beta_{n}\right) \rightarrow \alpha$ is defined by:

$$
\begin{aligned}
\hat{\varphi}\left(s(k),\left(y_{1}, \cdots, x, \cdots, y_{n}\right)\right) & =\varphi\left(y_{1}, \cdots, \hat{\varphi}\left(k,\left(y_{1}, \cdots, x, \cdots, y_{n}\right)\right), \cdots, y_{n}\right) \\
\hat{\varphi}\left(0,\left(y_{1}, \cdots, x, \cdots, y_{n}\right)\right) & =x
\end{aligned}
$$

\section{Proposition 2.}

$\hat{\varphi}\left(k,\left(y_{1}, \cdots, \varphi\left(y_{1}, \cdots, y, \cdots, y_{n}\right), \cdots, y_{n}\right)\right)=\varphi\left(y_{1}, \cdots, \hat{\varphi}\left(k,\left(y_{1}, \cdots, y, \cdots, y_{n}\right)\right), \cdots, y_{n}\right)$

Proof. By induction on $k$.

An immediale consequence of Definition 1 is $\hat{\varphi}(1, x)=\varphi(x)$, where $x: \beta_{1} * \cdots * \alpha *$ $\cdots * \beta_{n}$.

Having made the iteration structure of functions explicit, the next theorem helps program transformations exploit that structure. To simplify the exposition, consider the case in which $\varphi: \alpha \rightarrow \alpha$. In this case $\hat{\varphi}: N a t * \alpha \rightarrow \alpha$ and $\hat{\varphi}(k, n)=\varphi^{k}(x)$, where $\varphi^{k}$ denotes $k$ applications of $\varphi$. Suppose now that $f: N a t * N a t \rightarrow \alpha$ satisfies the equation: $f(s(i), s(n))=\varphi(f(i, n))$; then $f(4,7)=\varphi^{4}(f(0,3))=\hat{\varphi}(4, f(0,3))$. More generally, $f(i+k, n+k)=\hat{\varphi}(k, f(i, n))$, which is the result expressed by Theorem 3 . 
Theorem 3. Assume $f$ of type $N a l^{n} \rightarrow \alpha$, let $y_{i}$ be a term of type $\beta_{i}$ for each $i=1, \cdots, n$, and let $\varphi$ be a function of type $\beta_{1} * \cdots * \alpha * \cdots * \beta_{n} \rightarrow \alpha$. The following are equivalent:
1. $f\left(s\left(x_{1}\right), \cdots, s\left(x_{n}\right)\right)=\varphi\left(y_{1}, \cdots, f\left(x_{1}, \cdots, x_{n}\right), \cdots, y_{m}\right)$
2. $\hat{\varphi}\left(k,\left(y_{1}, \cdots, f\left(x_{1}, \cdots, x_{n}\right), \cdots, y_{n}\right)\right)=f\left(x_{1}+k, \cdots, x_{n}+k\right)$

Proof. That 1 implies 2 is obvious by instantiating $k$ to 1 . The converse is proved by induction on $k$.

To apply this theorem to $(5)$, let $M w p O(x)$ be $M w p(0, x)$ and introduce the equation: $\left.\widehat{M w p} O(k, \sigma(i, n)))=\sigma_{i}^{(} i+k, n+k\right)$. This gives the equational definition of $\sigma(i, n)$ :

$$
\begin{aligned}
& \sigma(s(i)+k, k)=\widehat{M w p} 0(k, u n i t(0)) \\
& \sigma(k, k)=\widehat{M w p} 0(k, A b s(A p p(\operatorname{Var}(0), \operatorname{Var}(1)))) \\
& \sigma(k, s(n)+k)=\widehat{\operatorname{Mwp}} 0(k, \operatorname{unit}(n))
\end{aligned}
$$

This definition can be rewritten in the conditional form described at the beginning of the section with $e_{1}=\widehat{M w p} O(n, \operatorname{unit}(0)), e_{2}=\widehat{M w p} O(i, \operatorname{Abs}(\operatorname{App}(\operatorname{Var}(0), \operatorname{Var}(1))))$ and $e_{3}=\widehat{M w p} 0(i$, unit $(n-i-1))$.

\subsection{Second Transformation Step}

The second transformation step transforms the expressions $e_{1}, e_{2}$ and $e_{3}$. The definition of $\widehat{M w p} 0$ of type Term $\rightarrow$ Term, obtained by Definition 1, refers to the (inefficient) function $M w p 0$. To get an efficient program an alternative (but equivalent) definition of $\widehat{M w p} 0$ that does not refer to $M w p O$ must be generated. Theorem 4 addresses this issue.

To introduce Theorem 4, consider the function upto. Informally, upto $(i, n)=$ $[i, i+1, \cdots, n]$. The function upto satisfies upto $(s(i), s(n))=\operatorname{map} s$ upto $(i, n)$. Let map_s be the specialization of the definition of map by $s$ :

$$
\begin{aligned}
& \text { map_s }[]=0 \\
& \text { map_s }(x:: x s)=s(x)::(\text { map_s } x s)
\end{aligned}
$$

The operators [ and :: are the constructors of the data type Lisl $(\alpha)$. By Theorem 3,

$$
(\widehat{\operatorname{map} s})(k, u p t o(i, n))=(\text { map_s })^{k}(u p t o(i, n))=u p t o(i+k, n+k)
$$

Theorem 4 will yield the following recursive definition of $\left(\right.$ map_s) ${ }^{k}$, (that is of $\widehat{\operatorname{map} \_s}$ ); it does not refer to map_s.

$$
\begin{aligned}
& \left(m a p_{-} s\right)^{k}[=[ \\
& \left(m a p_{-} s\right)^{k}(x:: x s)=s^{k}(x)::\left((\text { map_s })^{k} x s\right)
\end{aligned}
$$

Note, in this definition (mapss) ${ }^{k}$ is the function being defined. It is to be regarded atomically; map_s is neither defined nor referred to. 
Theorem 4. Let $y_{i}$ be a term of type $\beta_{i}$ for each $i=1, \cdots, n$, let $\varphi$ be a function of type $\beta_{1} * \cdots * \alpha * \cdots * \beta_{n} \rightarrow \alpha$, and let $C$ be a constructor of type $\alpha$. The following are equivalent:

1. $\varphi\left(y_{1}, \cdots, C\left(x_{1}, \cdots, x_{n}\right), \cdots, y_{n}\right)=C\left(\varphi_{1}\left(x_{1}\right), \cdots, \varphi_{n}\left(x_{n}\right)\right)$

2. $\hat{\varphi}\left(k,\left(y_{1}, \cdots, C\left(x_{1}, \cdots, x_{n}\right), \cdots, y_{n}\right)\right)=C\left(\widehat{\varphi_{1}}\left(k, x_{1}\right), \cdots, \widehat{\varphi_{n}}\left(k, x_{n}\right)\right)$

Proof. That 1 implies 2 is obvious by instanciating $k$ to 1 . The converse is proved by induction on $k$.

If $\mathrm{C}$ is a constructor of arity zero, Theorem 4 degenerates to the two equations $\varphi\left(y_{1}, \cdots, C, \cdots, y_{n}\right)=C$ and $\ddot{\varphi}\left(k,\left(y_{1}, \cdots, C, \cdots, y_{n}\right)\right)=C$.

To apply this result to $\widehat{N w p} 0$, recall that $M w p O(x)=M w p(0, x)$ and that:

$$
\begin{aligned}
\operatorname{Mwp}(i, \operatorname{Var}(n)) & =\operatorname{Var}(f(i, n)) \\
\operatorname{Mwp}(i, \operatorname{Abs}(t)) & =\operatorname{Als}(\operatorname{Mwp}(s(i), t)) \\
\operatorname{Mwp}\left(i, A p p\left(t, t^{\prime}\right)\right) & =\operatorname{App}\left(\operatorname{Mwp}(i, t), \operatorname{Mwp}\left(i, t^{\prime}\right)\right) .
\end{aligned}
$$

Introduction of the specializations $f_{0}(x)=f(0, x)$, and $M w p f(x)=M w p(1, x)$ allows the application of Theoren 4, producing:

$$
\begin{aligned}
& \widehat{\operatorname{Mup}} 0(k, \operatorname{Var}(n))=\operatorname{Var}\left(\widehat{f}_{0}(k, n)\right) \\
& \widehat{\operatorname{Mwp}} O(k, A b s(t))=A b s(\widehat{M w p} I(k, t)) \\
& \widehat{M w p} O(k, A p p(s, t))=A p p(\widehat{M u p} O(k, s), \widehat{M w p} O(k, t)) \text {. }
\end{aligned}
$$

It is easy to show that $\widehat{f}_{0}=\hat{s}$ because $f(0, x)=s(x)$, and that $\hat{s}(k, a)=a+k$ by induction on $k$. Therefore $\widehat{\operatorname{Mwp}} O(k, \operatorname{Var}(n))=\operatorname{Var}\left(\hat{f}_{0}(k, n)\right)$, which is equivalent to $\operatorname{Var}(\hat{s}(k, n))$, which can be rewritten $\operatorname{Var}(n+k)$. Although this appears to have progressed, it is incomplete because $\widehat{M w p} I$ is still defined in terms of $M w p 1$. Attempts to define $\widehat{A w p} 1$ by this method, however, will require the function $\widehat{M w p}$; this would continue forever. Fortunately, there is another way in which Theorem 3 may be applied to (5), yielding the equation $\widehat{M w} p(k,(0, \sigma(i, n)))=\sigma(i+k, n+k)$. Applying the same transformation as above produces another conditional definition of $\sigma(i, n)$ with $e_{1}=$ unil $(n), e_{2}=\widehat{\mid I w p}(i,(0, A b s(A p p(\operatorname{Var}(0), \operatorname{Var}(1)))))$ and $e_{3}=u n i t(n-1)$. Application of Theorem 4 produces a recursive definition of $\widehat{M f w}$ that does not refer to $M w p$ :

$$
\begin{aligned}
& \widehat{\operatorname{Mwp}}(k,(i, \operatorname{Var}(n)))=\operatorname{Var}(\widehat{f}(k,(i, n))) \\
& \widehat{\operatorname{Mwp}}(k,(i, A p p(s, t)))=\operatorname{App}(\widehat{\operatorname{Mwp}}(k,(i, s)), \widehat{\operatorname{Mwp}}(k,(i, t))) \\
& \widehat{\operatorname{Mwp}}(k,(i, A b s(t)))=\operatorname{Abs}(\widehat{\operatorname{Mwp}}(k,(s(i), t)))
\end{aligned}
$$

The transformation is not yet finished. Equation (8) remains to be improved by finding a recursive definition of $\hat{f}$ that does not refer to the function $f$. 


\subsection{Transformation of $\hat{f}$}

Recall the equations for $f$ :

$$
\begin{aligned}
& f(0, n)=s(n) \\
& f(s(i), 0)=0 \\
& f(s(i), s(n))=s(f(i, n))
\end{aligned}
$$

Applying Theorem 4 to (11) yields:

$$
\hat{f}(k,(s(i), s(n)))=s(\hat{f}(k,(i, n))) .
$$

This suggests attempting a conditional definition for $\hat{f}$. Using equations (9), (10), (11), Theorem 4, Theorem 3, and Definition 1 produces:

$$
\begin{aligned}
& \hat{f}(k,(0, s(n)))=s(\hat{s}(k, n))=s(n+k) \\
& f(k,(s(i), 0))=0 \\
& \hat{f}(k,(0,0))=k
\end{aligned}
$$

Applying Theorem 3 เo (12) gives: $\hat{f}(k,(i+p, n+p))=\hat{s}(p, \hat{f}(k,(i, n)))=\hat{f}(k,(i, n))+$ p. Applying that to equations (13), (14), (15) produces

$$
\begin{aligned}
& \hat{f}(k,(s(i)+p, p))=p \\
& \hat{f}(k,(p, s(n)+p))=n+1+k+p \\
& \hat{f}(k,(p, p))=k+p
\end{aligned}
$$

This equational definition is equivalent to the program:

$$
\hat{f}(k,(i, n)):=\text { if } i>n \text { then } n \text { clse if } i=n \text { then } n+k \text { clse } n+k .
$$

The program simplifies to: $\hat{f}(k,(i, n))=$ if $i>n$ then $n$ else $n+k$. By unfolding $\hat{f}$ and by a wel known property of the conditional, equation (8) becomes: $\widehat{M w p}(k,(i, \operatorname{Var}(n)))==$ if $i>n$ then $\operatorname{Var}(n)$ else $\operatorname{Var}(n+k)$. Including the transformed form of $\sigma$, which comes from above, produces the program in Fig. 4 which does not perform redundant computations for $\sigma_{i}$ and $f_{i}$. The transformation involved in this section has been done manually. However the transformation process is systematic and involves equational reasoning using Theorem 3 and Theorem 4 . It shows implicitly how to automatically transform a function of type $\mathrm{Nat} * \mathrm{Nat} \rightarrow \mathrm{Nat}$ into a more efficient conditional form.

\section{Directions}

The paper has presented a clearly molivated and correct specification for a subtle representation of $\lambda$-terms, the implementation of which has, in the second authors experience, been prone to "off by one" errors. It has taken this abstract specification, with its extensive use of higher-order concepts, reduced it to a first-order program, 


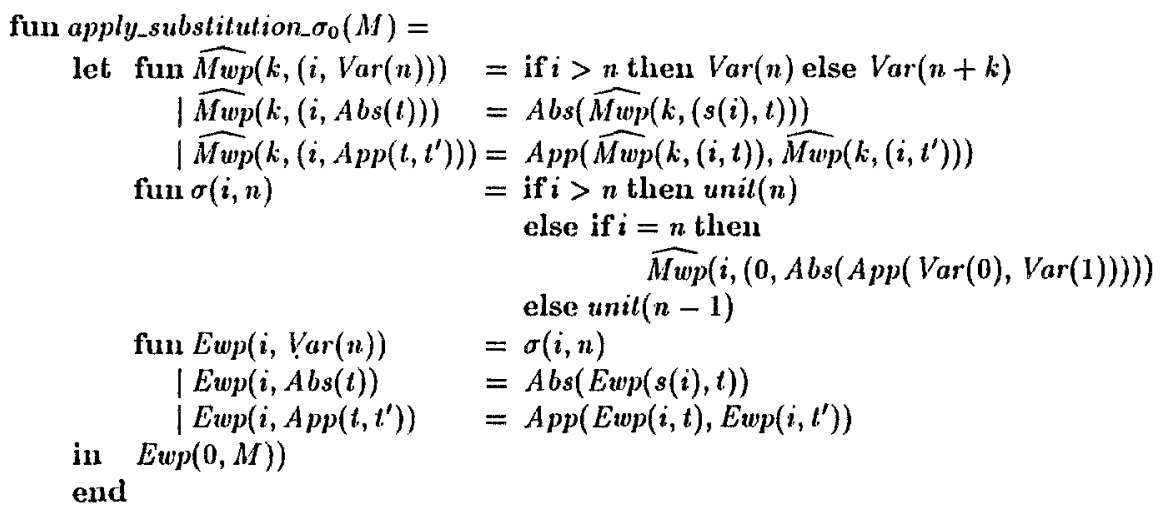

Fig. 4. Final result

introduced index arithmetic and produced an efficient algorithm that exploits computer arithmetic.

This development, illustrates several new techniques. First, it makes the monadic structure in the development of the specification explicit by showing that it is a monad in FUNSEQ. It supports this structure will new program transformation techniques which allow the implicit use of arithmelic to be "rediscovered" formally. Finally, it demonstrates the feasibility of integrating tools for monadic programming and specification, which tend to be higher-order, with relatively standard program transformation technology, which is strictly first-order. The importance of partial evaluation technology in bridging this gap cannot be overstated.

\subsection{Technology}

Currently our technology is a tower of Babel. Automatic support for monadic programming, including automatic program generation, exists in CRML, a Standard ML derivative developed by Sheard. The partial evaluator, Schism, uses its own (typed) dialect of Scheme as its object language. Astre, Bellegarde's program transformation system, is written in CAML. It uses a very simple first-order language as its object language.

In this cnviromment, claims that the development is automatable mean that we have automated the process "piccewise", translating between the formalisms in a nearly mechanical fashion. It is, of course, our vision that one day these tools will all work in concert, allowing a development to proceed from specification to efficient realization with human intervention only when necessary.

\section{$7.2 \quad$ Reuse}

Although this paper has focused on the $\lambda$-calculus, the specification can be applied to virtually any abstract syntax with a regular binding structure provided its lype 
can be expressed as a monad and the appropriate definition of map_with_policy can be given. For example, adding boolean constants and a conditional has no effect on the specification of substitution and only changes map_with_policy by defining it. to apply $f$ recursively on the components of the conditional without applying $Z$. Adding let is also trivial; again, no changes need to be made to the specification of substitution-only to map_with_policy. In this case, map_with_policy must apply $Z$ to $f$ when it enters the component in which the bound variable has been introduced. This ability to reuse specifications is one of the strongest arguments for the adoption of monads as a tool to structure program specification and development.

But what about the transformations? Can we reuse program improvements? Here we have less experience, however the decisions that are required to improve programs for the different scenarios outlined above are substantially the same. It appears that a transformation system that records its development may be able to replay the development and obtain similar improvements.

\section{References}

1. Françoise Bellegarde. Program transformation and rewriting. In Proceedings of the fourth conference on Rewriting Technigues and Applications, volume 488 of LNCS, pages 226-239, Berlin, 1991. Springer-Verlag.

2. N. G. de Bruijn. Lambda calculus notation with nameless dummies, a tool for automatic formula manipulation, with application to the Church-Rosser theorem. Indagaciones Mallemalische, 34:381-392, 1972.

3. N. G. de Bruijn. Lambda calculus with namefrce formulas involving symbols that represent reference transforming mappings. In Proc. of the Koninklijke Nederlandse Akademie van Welenschappen, pages 348-356, Amsterdam, series A, volume 81(3), September 1978.

4. Charles Consel. The Schism Manual. Technical report, Oregon Grad. Inst., 1992.

5. James Hook, Richard Kieburtz, and Tim Sheard. Generating programs by reflection. Technical Report 92-015, Oregon Grad. Inst., July 1992.

6. Eugenio Moggi. Notions of computations and monads. Information and Computation, 93(1):55-92, July 1991.

7. John C. Reynolds. Definitional interpreters for higher-order programming languages. In $A C M$ National Conference, pages $717-740$. ACM, 1972.

8. Philip Wadler. The essence of lunctional programming. In POPL '92. ACM Press, January 1992. 